\title{
Mitteilungen
}

\section{Tox Info Suisse}

\section{Vergiftungen: mehr als 41000 Beratungen}

Tox Info Suisse führte 2018 mit 41151 rund 800 Beratungen zu Vergiftungen mehr durch als im Vorjahr. Die telefonische Notfallberatung unter der Nummer 145, auch via Tox Info App, steht Laien und Fachpersonen rund um die Uhr zur Verfügung. Die ersten Analysen der Beratungstätigkeit werden hier vorgestellt. Eine besondere Zunahme zeigten Anfragen zu Tiervergiftungen und Beratungen für Tierärzte.

Mit 41151 Anrufen wurde die Giftberatung gegenüber dem Vorjahr 2,1\% häufiger in
Anspruch genommen, was dem Trend der letzten Jahre entspricht (Abb. 1). 93\% der Anfragen erfolgten aufgrund eines Giftkontaktes, während $7 \%$ vorsorglicher Natur waren. In zwei Dritteln der Fälle wurden die Betroffenen oder ihre Angehörigen direkt beraten. In den übrigen Fällen fragten Fachpersonen oder Institutionen und Organisationen nach speziellen Informationen zu Risikoabschätzung und Massnahmen bei akuten Intoxikationen. Von den Vergiftungen waren vor allem Kinder betroffen (55\%), davon $85 \%$ im Vorschulalter. Intoxikationen mit Medikamenten, Haushaltprodukten und Pflanzen machten $70 \%$ aller Vergiftungsfälle aus (Tab. 1). Bei Kindern

Tabelle 1: Beratungen von Tox Info Suisse 2018, nach Häufigkeit der Giftstoffgruppen bei Vergiftungen beim Menschen* (Prozent aller Fälle, $n=33394$ ).

\begin{tabular}{ll}
\hline Medikamente & $34,8 \%$ \\
\hline Haushaltprodukte & $25,7 \%$ \\
\hline Pflanzen & $9,3 \%$ \\
\hline Produkte für Körperpflege und Kosmetika & $6,8 \%$ \\
\hline Technisch-gewerbliche Produkte & $6,4 \%$ \\
\hline Nahrungsmittel und Getränke & $4,8 \%$ \\
\hline Genussmittel, Drogen und Alkohol & $3,3 \%$ \\
\hline Produkte in Landwirtschaft und Gartenbau & $2,3 \%$ \\
\hline Pilze & $1,7 \%$ \\
\hline Gifttiere & $1,3 \%$ \\
\hline Andere/Unbekannte & $3,4 \%$ \\
\hline
\end{tabular}

* Nicht berücksichtigt sind hier Beratungen zu Vergiftungen bei Tieren sowie theoretische Anfragen ohne Exposition (ohne Substanzkontakt).

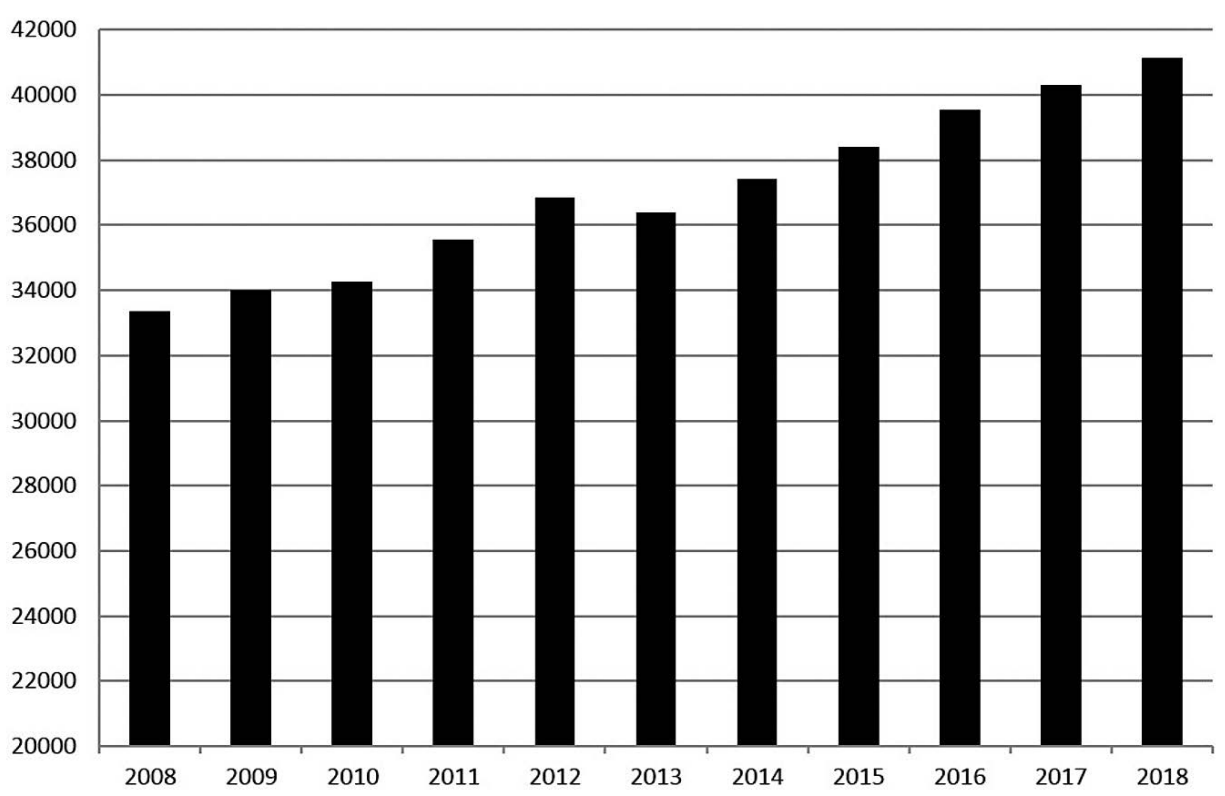

Abbildung 1: Anzahl Anfragen an Tox Info Suisse in den letzten zehn Jahren.

handelt es sich dabei typischerweise um Unfälle, bei den Erwachsenen stehen die beabsichtigten Selbstvergiftungen im Vordergrund, vor allem Selbstmordversuche (65\%) und der Substanzmissbrauch (15\%).

In den letzten Jahren kam es zu einer laufenden Zunahme der Beratungen zu Tiervergiftungen (2180). Betroffen waren vor allem Hunde (1630-mal) und Katzen (409mal), aber auch Pferde und Ponys (60), Hasen und Kaninchen (35), Schafe oder Ziegen (15); andere Tierarten waren seltener. In etwas mehr als der Hälfte der Fälle fragten die Tierhalter um Rat, in den übrigen Fällen wurden Tierärztinnen und -ärzte beraten. Die Zahlen zeigen, dass Hunde besonders gefährdet sind, da sie dazu neigen, alles zu verschlingen, was sie finden.

Zum Verlauf und zum Schweregrad der Vergiftungen gibt der Jahresbericht von Tox Info Suisse Auskunft, der im August 2019 erscheinen wird.

Diese und weitere Neuigkeiten zu aktuellen Themen rund um Gifte und Vergiftungen erhalten Sie durch unsere Tox Info App. Zusätzliche Informationen zu Giften und Vergiftungen sind auf der Website von Tox Info Suisse zu finden (www.toxinfo.ch).

Weitere Auskünfte: Dr. med. Hugo Kupferschmidt, Direktor Stiftung Tox Info Suisse, Freiestrasse 16, CH-8032 Zürich Tel.+41 4425166 66, Fax +41 442528833 E-Mail: info[at]toxinfo.ch

Swiss Association for Laparoscopic and Thoracoscopic Surgery SALTS

\section{Innovation Grant}

The Swiss Association for Laparoscopic and Thoracoscopic Surgery SALTS awards a grant of CHF 5000 for an innovative clinical project taking advantage of a minimally invasive approach.

Requirements: Innovative clinical project capitalizing on a minimally invasive approach with a sound scientific approach. Swiss authors can submit work done abroad, non-Swiss authors must have performed their project in Switzerland. Electronic submission (pdf or word). Language: English, French or German.

\section{Reception deadline: April 30th, 2019}

Submission to: PD Dr. N. Buchs, Scientific Officer SALTS, Hôpitaux universitaires de 
Genève, Département de chirurgie, Rue Gabrielle-Perret-Gentil 4, CH-1205 Genève, nicolas.c.buchs[at]hcuge.ch

Swiss Association for Laparoscopic and Thoracoscopic Surgery SALTS

\section{Zollikofer Award}

The Swiss Association for Laparoscopic and Thoracoscopic Surgery SALTS strongly supports research in laparoscopic and thoracoscopic surgery. The Association invites researchers to apply for the Zollikofer Award of the year 2019. The amount of the Award is CHF 3000.

Topic: Basic science and clinical investigation in laparoscopic and thoracoscopic surgery.

Requirements: Original article published or accepted for publication in a peer-reviewed journal in 2018 or 2019. Swiss authors can submit work done abroad, non-Swiss authors must have performed their research in Switzerland. Electronic submission (pdf or word) including proof of acceptance if the paper is not yet indexed in Pubmed. Language: English, French or German.

Reception deadline: April 30th, 2019

Submission to: PD Dr. N. Buchs, Scientific Officer SALTS, Hôpitaux universitaires de Genève, Département de chirurgie, Rue Gabrielle-Perret-Gentil 4, CH-1205 Genève, nicolas.c.buchs[at]hcuge.ch
Schweizerische Gesellschaft für Chirurgie

Preis der Schweizerischen Gesellschaft für Chirurgie 2019

Zur Förderung der Chirurgischen Forschung vergibt die Schweizerische Gesellschaft für Chirurgie (SGC) einen Preis für wissenschaftliche Arbeiten aus der chirurgischen Forschung. Die Richtlinien in englischer Sprache sind auf der SGC Homepage unter «Preise der SGC» abrufbar.

Folgende Bedingungen müssen erfüllt sein: - Erstautor muss Chirurg/-in und unter 40 Jahren alt sein;

- Originalarbeit (zur Publikation akzeptiert oder vor kurzem publiziert mit peer review) oder angenommene Habilitationsschrift;

- zugrunde liegende Untersuchungen mehrheitlich in der Schweiz durchgeführt.

Das Preisgeld beträgt $10000 \mathrm{CHF}$, wobei der Preis je nach Juryentscheid eventuell geteilt werden kann.

Bewerber/-innen werden gebeten, die Unterlagen in einer pdf-Datei und unter Angabe der Zeitschrift inklusive dem "Impact Factor", in welcher die Arbeit publiziert wurde oder wird, bis zum 15. Februar 2019 an die Mail-Adresse der SGC/SSC-Geschäftsstelle: info[at]sgc-ssc.ch zu senden.

\section{gynécologie suisse SGGG \\ SGGG-Bayer-Forschungs-Grant für Nachwuchsforschende}

Die gynécologie suisse SGGG schreibt 2019 erstmals den SGGG-Bayer-Forschungs-Grant aus. Der Grant ist mit 80000 Franken dotiert und soll alle zwei Jahre vergeben werden. Er richtet sich an Ärztinnen und Ärzte in der Schweiz, welche sich in Weiterbildung zum Facharzttitel Gynäkologie und Geburtshilfe befinden oder diesen bereits haben und eine wissenschaftliche/akademische Karriere anstreben. Sie sollen das 40. Altersjahr noch nicht überschritten und sich bereits durch wissenschaftliche Leistungen (Nachweis bereits erbrachter Leistungen in der Forschung) ausgezeichnet haben. Bereits Habilitierte sind nicht zugelassen.

Dieser Forschungs-Grant soll explizit für die Förderung einzelner Personen eingesetzt werden und diesen ermöglichen, Freiräume für die eigene Forschungstätigkeit zu schaffen. Er ist daher nicht als Projektförderung gedacht.

Der vollständige Ausschreibungstext und das Gesuchsformular sind auf der Homepage unter www.sggg.ch abrufbar.

Der Antrag muss bis am 15. März 2019 beim Sekretariat der SGGG, Altenbergstrasse 29, Postfach 686, 3000 Bern 8, eingereicht werden.

\section{Hilfskasse für Schweizer Ärzte \\ Eingegangene Spenden vom 1.10.2018 bis 31.12.2018}

Vom 1. Oktober bis 31. Dezember 2018 sind 26 Spenden im Gesamtbetrag von 4665 Franken eingegangen.

Der Stiftungsrat der Hilfskasse für Schweizer Ärzte freut sich sehr, diese Gaben bekanntgeben zu dürfen und dankt allen Spendern recht herzlich.

Damit die Spenden in voller Höhe den Destinatären zukommen, haben wir uns entschlossen, für Spenden unter 500 Franken auf den Versand von persönlichen Dankschreiben zu verzichten. Wir hoffen sehr, dass diese Massnahme bei allen Spendern auf Verständnis stösst.

Für die Hilfskasse für Schweizer Ärzte Der Kassier des Stiftungsrates 